\title{
Fatores do absenteísmo em primeira consulta num ambulatório de oncologia em um Hospital Universitário
}

\author{
Factors of absenteeism in first consultation in an oncology outpatient clinic in a \\ University Hospital
}

Túlio de Oliveira Mazza1 ${ }^{1}$ Gabriela Souza Assis Ferreira², Renato Mantelli Picoli², André Lucirton Costa ${ }^{3}$

\begin{abstract}
RESUMO
Introdução: o absenteísmo dos usuários de sistemas de saúde representa um grande problema para as organizações. O objetivo deste estudo foi identificar as razões para o absenteísmo dos pacientes em consulta médicas. Metodologia: pesquisa de campo nos ambulatórios de oncologia do Hospital das Clínicas da Faculdade de Medicina de Ribeirão Preto. Os pacientes que não compareceram à primeira consulta em 2013 (356) foram contatados por meio telefônico, dos quais 299 não foi possível o contato por apresentarem telefone inválido, desligado, fora da área de cobertura ou por não atenderem a ligação. Resultados: 57 pacientes foram entrevistados e apontaram os motivos para falta. As respostas foram categorizadas. Os resultados mostraram que 27 pacientes (47\%) não compareceram à consulta por motivos relacionados ao indivíduo ou comportamento, sendo "consultei-me em outro lugar" o mais recorrente $(12,30 \%)$, seguido de "consulta coincidiu com outro compromisso" $(7,02 \%)$ e "não me lembro o motivo" (7,02\%). Individualmente, o motivo mais citado pelos pacientes foi "eu não faltei a consulta" (31,6\%), o que sugere problemas de comunicação. Conclusão: as principais razões para o absenteísmo estão relacionadas ao indivíduo e que problemas de cadastro do paciente e comunicação dificultam a compreensão das causas do absenteísmo.
\end{abstract}

Palavras-Chave: Absenteísmo. Mau Uso de Serviços de Saúde. Sistema Único de Saúde. Eficiência.

\section{ABSTRACT}

Introduction: absenteeism of health system users is a major problem for organizations. The study aimed to identify the factors of absenteeism in outpatients at the General Hospital of the Medical School of Ribeirão Preto oncology clinics. Methodology: patients who did not attend the first appointment in 2013 (356) were contacted by telephone, of which 299 were not able to contact because they presented

1. Graduado em Administração de Empresas pela Faculdade de Administração e Contabilidade de Ribeirão Preto - FEARP-USP

2. Doutorandos em Administração de Organizações pela Faculdade de Administração e Contabilidade de Ribeirão Preto - FEARP-USP

3. Docente da Faculdade de Administração e Contabilidade de Ribeirão Preto - FEARP-USP e Docente do curso de Mestrado Profissional de Gestão de Organizações de Saúde - FMRP-USP
CoRrespondênCIA: André Lucirton Costa Faculdade de Economia, Administração e Contabilidade de Ribeirão Preto, FEA-RP/USP

Avenida Bandeirantes, 3900 14040-905 - Ribeirão Preto/SP - Brasil alcosta@usp.br

Recebido em 08/08/2018 Aprovado em 30/01/2019 
an invalid telephone number, disconnected, outside the coverage area or because they did not answer the call. Results: 57 patients were interviewed and indicated reasons for no show. The answers were categorized. The results showed that 27 patients (47\%) did not attend the appointment for reasons related to the individual or behavior, the cause "I had an appointment elsewhere" was the most recurrent $(12.30 \%)$, followed by "the appointment happened at the same time of another commitment" $(7.02 \%)$ and "I cannot remember the reason" (7.02\%). Individually, the most cited reason by the patients was "I did not miss the appointment" 18 (31.6\%), thus suggesting communication problems. Conclusion: the main reasons for absenteeism are related to the individual and problems of patient registration and communication make it difficult to understand the causes of absenteeism.

Keywords: Absenteeism. Health Services Misuse. Unified Health System. Efficiency.

\section{INTRODUÇÃO}

O absenteísmo dos usuários dos sistemas de saúde representa um grande problema para as organizações, pois provoca a subutilização dos recursos empregados, com custo de oportunidade dos serviços e recursos destinados ao atendimento e requer esforço extra para reagendar a consulta, causando possível comprometimento da saúde dos usuários. ${ }^{1}$ Dessa forma, a ausência dos pacientes às consultas agendadas interfere no cuidado da saúde e gera desperdício de recursos médicos e administrativos, interferindo na eficiência dos sistemas de saúde e obstruindo tentativas de redução do tempo de espera, devendo as razões do não comparecimento dos pacientes às consultas serem analisadas, para buscar soluções e minimizar o problema..$^{1-5}$

Dentre as razões para o absenteísmo, o "esquecimento" é o principal motivo levantado pelos pacientes para justificar a ausência às consultas em atendimento primário no Reino Unido, como mostra estudo conduzido por um período de três semanas, no qual os principais motivos apresentados foram o esquecimento do compromisso e a dificuldade em cancelar a consulta. ${ }^{6}$ No entanto, estudo realizado na África do Sul identificou como razão principal para o absenteísmo os "problemas com transporte". ${ }^{7}$

Em Genebra, na Suíça, estudo realizado em um hospital universitário identificou que fatores locais podem ter uma importância significativa no absenteísmo. Com 1296 consultas agendadas, 206 pacientes não compareceram. Assim, o estudo identificou taxa de absenteísmo de 15,9\%. ${ }^{8}$

Em Lisboa, as faltas de adolescentes em consultas hospitalares, para o período de $30 \mathrm{me}-$ ses, apontou taxa de absenteísmo de $28,5 \%$, tendo como principais motivos relatados a incompa- tibilidade com os horários de aula, com 28,3\%, e o esquecimento representando $17 \%$ das causas de absenteísmo. ${ }^{9}$

Uma pesquisa em um hospital universitário na Arábia Saudita, mostrou um absenteísmo em consultas de $23,7 \%$. Os motivos mais frequentes apresentados para as faltas foram indisponibilidade de transporte $(22,6 \%)$ e esquecimento $(17,3 \%)$. Os autores concluíram que prover meios de transporte e enviar lembretes aos pacientes podem ajudar a reduzir o problema e que mais pesquisas sobre 0 assunto são necessárias. ${ }^{10}$

No Canadá, observou-se um absenteísmo de $24,4 \%$ em consultas agendadas no serviço público de saúde para especialidade de otorrinolaringologia. ${ }^{5}$ Nos Estados Unidos, os motivos mais frequentes para ausência em consultas em uma clínica de neurologia pediátrica foram de 25,9\% para "problemas ou conflitos de agenda" e 20,4\% para "esquecimento". ${ }^{11}$ Com isso, o "esquecimento" e o "não conhecimento sobre o agendamento" são as principais causas do não comparecimento aos compromissos nos serviços de saúde, razões para aproximadamente $40 \%$ das faltas. ${ }^{12}$

No Brasil, o absenteísmo dos usuários do Sistema Único de Saúde (SUS) é um problema com um índice médio de $25 \% .{ }^{13}$ Dentre as motivações para ausência dos usuários do SUS às consultas médicas e odontológicas estão os problemas com transporte e comunicação, bem como o esquecimento e o agendamento em horários inoportunos. ${ }^{14-17}$ Com $32 \%$ de faltas em agendamentos de consultas em atenção básica no município do Rio de Janeiro, estas ausências geram desperdício de recursos, falta de continuidade do tratamento, dano ao processo de trabalho e aumento da fila de espera. ${ }^{15}$ 
Considerando a organização do SUS, os níveis de atenção à saúde são estruturados em ordem crescente de densidade tecnológica: atenção primária à saúde, com menor densidade tecnológica; atenção secundária à saúde, com densidade tecnológica intermediária e, atenção terciária à saúde constituída pela maior densidade tecnológica. ${ }^{18}$ Dessa forma, a atenção primária é a porta de entrada do sistema público de saúde e o acesso à atenção secundária e terciária se dá por meio da regulação assistencial, executada pelas Secretarias Estaduais e Municipais de saúde, baseada em protocolos, classificação de risco e outros critérios de priorização como controle do nível de complexidade dos serviços para melhor adequar a oferta desses serviços à necessidade do usuário. ${ }^{19}$

O Hospital das Clínicas da Faculdade de Medicina de Ribeirão Preto (HCFMRP) faz parte da rede de atenção de saúde do SUS, contudo possui um número limitado de vagas disponíveis para o atendimento de novos pacientes, primeira consulta, ofertando número de consultas menor que o necessário para atender a demanda apresentada à Central de Agendamento de Consultas pelos municípios de sua região de abrangência. Ademais, parte das vagas ofertadas pelo HCFMRP é desperdiçada por decorrência do absenteísmo. Em Ribeirão Preto, estudo realizado na rede pública de saúde, identificou taxa global de absenteísmo de $32,2 \%$, sendo $42,7 \%$ na atenção básica e $17 \%$ no atendimento hospitalar. ${ }^{20}$

Para minimizar essas ausências são utilizadas estratégias para identificar as necessidades de atenção à saúde por meio da regulação médica e adoção de protocolos clínicos e de regulação. ${ }^{21-24}$ Estudo realizado nos Estados Unidos analisou a eficácia de um sistema de lembretes para reduzir o absenteísmo em um ambulatório multidisciplinar de uma universidade em Nova Jersey, onde foi comparada a taxa de absenteísmo em pacientes que receberam ligações de um funcionário para lembrar do compromisso; pacientes que receberam ligações automatizadas para lembrar do compromisso; e pacientes que não receberam nenhum lembrete. As taxas de absenteísmo encontradas foram $13,6 \%, 17,3 \%$ e $23,1 \%$, respectivamente, sendo $78 \%$ dos que a receberam lembrete de um funcionário e $72 \%$ dos que a receberam de forma automatizada consideram a ação útil. ${ }^{25}$

Portanto, para minimizar os danos do absenteísmo em serviços de saúde é preciso identificar as razões para o não comparecimento às consultas agendadas e, assim, desenvolver ações que combatam essas causas. Dessa forma, este estudo objetiva identificar e descrever os motivos para faltas às primeiras consultas num hospital universitário terciário.

\section{MATERIAL E MÉTODOS}

Para identificar as causas do absenteísmo em primeiras consultas ambulatoriais no serviço público de saúde foi analisado o não comparecimento ao compromisso agendado nos ambulatórios de oncologia do Hospital das Clínicas da Faculdade de Medicina de Ribeirão Preto da Universidade de São Paulo (HCFMRP-USP). O HCFMRP-USP é uma autarquia mantida pelo governo do estado de São Paulo, vinculada à Secretaria de Estado da Saúde, com a missão de desenvolver e praticar assistência, ensino e pesquisa em saúde, por meio da busca permanente da excelência, contribuindo para a melhoria da qualidade de vida da população. ${ }^{26}$

Nos ambulatórios, segmentados por especialidades, são atendidos pacientes oriundos da rede (primeira consulta) e pacientes em segmento no hospital (retorno). Como os maiores índices de absenteísmo são registrados na primeira consulta, selecionou-se como população-alvo deste estudo os pacientes que faltaram à primeira consulta durante o ano de 2013 nos ambulatórios de oncologia do HCFMRP-USP. Os ambulatórios oncológicos estão alocados em treze diferentes especialidades (Tabela 1). A composição da amostra foi obtida por meio de dados secundários extraídos do sistema de informação da instituição. Na Tabela 1 são apresentados ainda o número de consultas agendadas para pacientes novos no ano de 2013 bem como o número de faltas registrados no período. 
Tabela 1

Composição da população

\begin{tabular}{lccc}
\hline Especialidade & $\begin{array}{c}\text { Número de consultas } \\
\text { agendadas }\end{array}$ & Número de faltas & $\%$ de faltas \\
\hline Gastro cirurgia de fígado oncológica & 17 & 4 & 23,5 \\
Neuro cirurgia oncológica & 74 & 16 & 21,6 \\
Gastro cirurgia oncológica & 88 & 17 & 19,3 \\
Dermatologia oncológica & 747 & 124 & 16,6 \\
Oncologia pneumologia & 97 & 16 & 16,5 \\
Oncologia proctologia & 82 & 37 & 15,9 \\
Oncologia de cabeça e pescoço & 234 & 58 & 15,8 \\
Oncologia urológica & 593 & 44 & 9,8 \\
Mastologia oncológica & 558 & 21 & 7,9 \\
Oncologia ginecológica & 289 & 2 & 7,3 \\
Oncologia geral & 28 & 15 & 7,1 \\
Oncologia ortopedia & 262 & 3 & 5,7 \\
Oncologia pediátrica & 68 & 370 & 4,4 \\
\hline Total & 3137 & 11,8 \\
\hline
\end{tabular}

Inicialmente, utilizou-se a técnica de amostragem estratificada proporcional, em que o tamanho da amostra extraída de cada estrato é proporcional ao tamanho relativo do estrato na população total. ${ }^{27,}{ }^{28}$ Assim, calculou-se o tamanho da amostra considerando a população como um todo, e posteriormente foi realizada a proporcionalidade nos estratos. Essa adaptação foi necessária, pois o cálculo do tamanho da amostra dentro de cada estrato, dado o universo pesquisado, tornaria tecnicamente inapropriado, dado que alguns deles possuem $n<30$. A fórmula utilizada para determinação do tamanho da amostra foi a do erro padrão da proporção para populações finitas. ${ }^{29}$

$$
n=\frac{z^{2} p(1-p) N}{(N-1) e^{2}+z^{2} p(1-p)}
$$

Considerando o tamanho da população (N) igual a 370 , a estimativa da proporção igual a $11,8 \%$, o erro de amostragem igual a $10 \%$ e a um nível de confiança de $99 \%$, temos $n=58,3$ como o tamanho da amostra, número de entrevistas necessárias para assegurar a precisão e a confiança da análise.

Entrevistas telefônicas apresentam uma taxa de resposta entre 60 e $80 \%$. Taxas maiores são obtidas por tentativas subsequentes de cha- madas. Muitas entrevistas realizadas por telefone tentam-se chamar de volta pelo menos três vezes. $^{30}$ Neste estudo foram realizadas pelo menos três tentativas de contato telefônico com cada paciente. Considerando uma taxa de resposta de $70 \%$, a amostra deve contar com 84 pacientes, assim a amostragem estratificada proporcional resultou em tamanhos de amostra diferentes para cada especialidade: Gastro cirurgia de fígado oncológica (1); Neuro cirurgia oncológica (4); Gastro cirurgia oncológica (4); Dermatologia oncológica (29); Oncologia pneumologia (4); Oncologia proctologia (4); Oncologia proctologia (3); Oncologia de cabeça e pescoço; Oncologia urológica (14); Mastologia oncológica (10); Oncologia ginecológica (5); Oncologia geral (1); Oncologia ortopédica (4) Oncologia pediátrica (1).

A amostragem em cada estrato se deu pelo processo de amostragem aleatória simples para assegurar a representatividade de todos os estratos na composição da amostra. A relação dos pacientes faltosos e os contatos telefônicos dos pacientes foram obtidos por meio de um relatório do cadastro de pacientes, extraído do sistema de informação do HCFMRP-USP. A pesquisa foi aprovada pelo Comitê de Ética em Pesquisa da Faculdade de Filosofia, Ciências e Letras de Ribeirão Preto (CAAE n. ${ }^{\circ}$ 31103714.2.0000.5407).

Para analisar o absenteísmo dos pacientes em novas consultas foram realizadas entrevistas 
por telefone no segundo semestre de 2014 com os pacientes que faltaram às consultas agendadas no ano de 2013. O entrevistado foi questionado sobre o motivo que o levou a não comparecer à consulta agendada, sendo a abordagem feita por meio de uma pergunta aberta categorizável, realizada após a leitura do Termo de Consentimento Livre e Esclarecido e anuência verbal do paciente.

As respostas foram analisadas e categorizadas segundo os principais motivos para o não comparecimento às consultas: esquecimento; trabalho; família; falta de transporte; estar muito doente para conseguir atender à consulta; desaparecimento dos sintomas; condições climáticas entre outros menos frequentes. $6,9,10,31,32,33$ Com isso, as respostas foram agrupadas em seis categorias: (1) indivíduo/comportamento; (2) família; (3) trabalho; (4) transporte; (5) clima e (6) outros. Em seguida foi realizada a análise dos dados por meio de estatística descritiva.

\section{RESULTADOS E DISCUSSÕES}

No HCFMRP-USP o agendamento das consultas eletivas para novos pacientes é feito pelo gestor Estadual, através da Central de Agendamento de Consultas, instalada no Departamento Regional de Saúde XIII, órgão estadual que tem a atribuição de regular os encaminhamentos de novos pacientes ao HCFMRP-USP. O processo de agendamento de novos pacientes bem como as organizações envolvidas são apresentados na Figura 1.

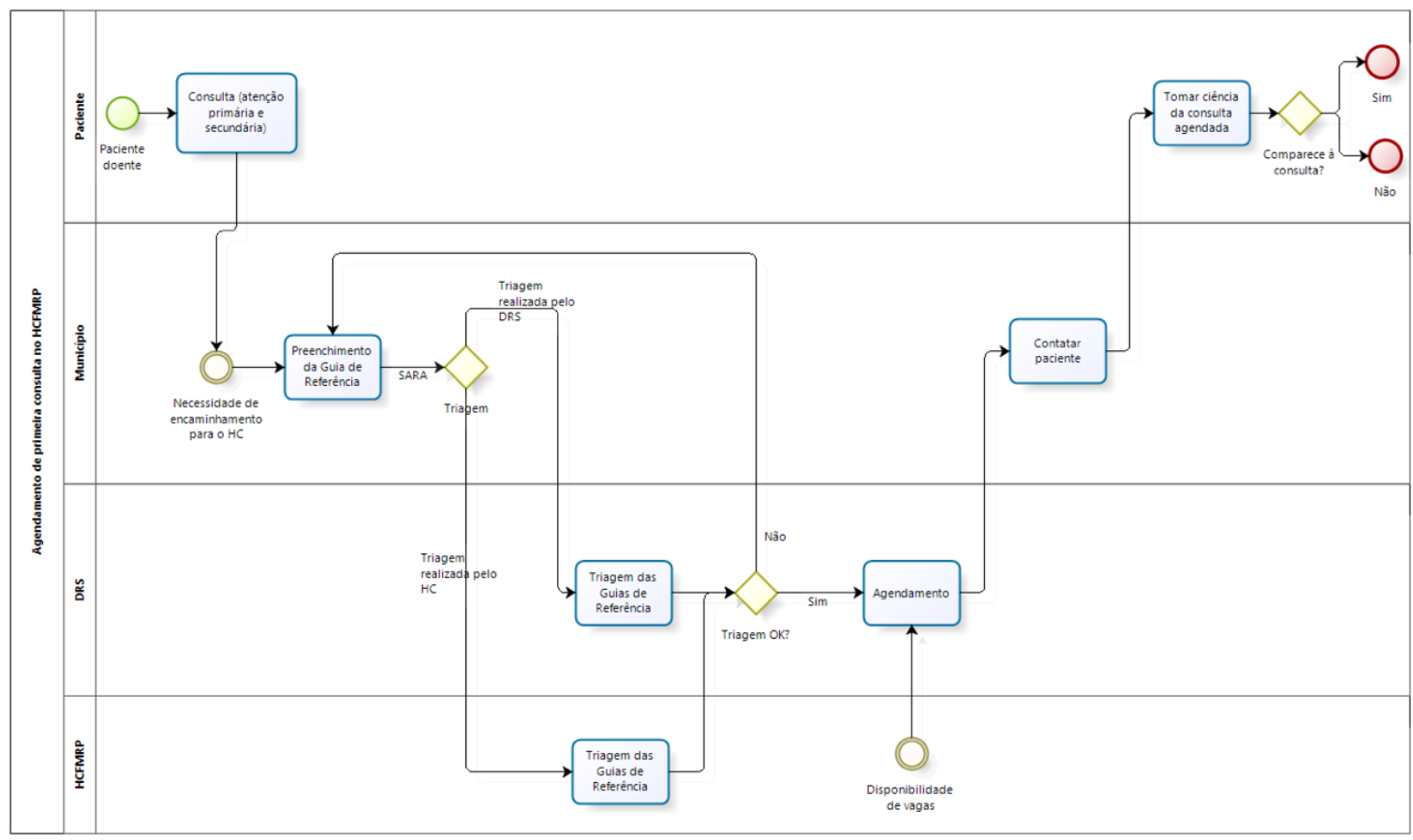

Figura 1: Desenho do processo de agendamento de consultas no HCFMRP.

O processo inicia-se quando um paciente é atendido na atenção básica no seu município e é identificada a necessidade de atendimento especializado de nível terciário. O usuário pode ser referenciado diretamente da atenção básica ou a partir da atenção secundária à saúde para o HCFMRP-USP.
O pedido de referência é formalizado por meio da Guia de Referência, onde constam as informações fornecidas pelo solicitante médico: dados do paciente; especialidade a que se destina; nível de prioridade, história pregressa; quadro clínico; diagnóstico ou hipótese diagnóstica; exames realizados; e demais informação julgadas necessária pelo médico 
em atendimento. A Guia de Referência é inserida no Sistema de Apoio a Regulação Assistencial (SARA), um sistema de informação que permite que as guias sejam digitais e integra municípios do estado de São Paulo, Departamentos Regionais de Saúde (DRS) e o HCFMRP-USP. Em seguida, é realizado o processo de avaliação das guias de referência denominado de triagem das guias de referência.

$\mathrm{Na}$ triagem, as informações são verificadas com base nos protocolos do HCFMRP-USP, considerando o correto preenchimento; informações pendentes ou faltantes; necessidade real a ser referenciado para à atenção terciária; prioridade correta; e especialidade de destino correta. Caso haja inconsistência na guia, essa retorna ao Município solicitante para que seja efetuada a correção ou complementação. Quando os dados e o encaminhamento são julgados como corretos, o paciente entra para a fila de agendamento do Estado, conforme os critérios de priorização.

O agendamento da consulta depende da disponibilidade de vagas no HCFMRP-USP, informado pelo Sistema Integrado de Informação Hospitalar (SIIH), interligado ao SARA. Quando há vagas disponíveis, o sistema as libera com antecedência de 30 dias para as DRS's, permitindo o agendamento a ser realizado pelo departamento baseado nas guias avaliadas. Realizado o agendamento, automaticamente é enviado ao Município o comprovante de confirmação de consulta, que consta as informações de data, horário, e balcão, sendo de responsabilidade do Município o contato com o paciente para informá-lo sobre o agendamento.

No decorrer das entrevistas foram observadas falhas no cadastro dos pacientes como dados desatualizados, número de telefone incorreto e cadastro incompleto. De um total de 356 pacientes, 154 não possuíam número de telefone válido cadastrado; 115 pacientes não atenderam a ligação ou o celular estava desligado/fora de área, ocorrido mesmo sendo realizadas três tentativas em horários diferentes; 30 pacientes haviam falecido (Tabela 2).

Dessa forma, foram efetivamente entrevistados 57 pacientes que relataram os motivos para o não comparecimento às consultas, descritos na Tabela 3.

\section{Tabela 2}

Característica da amostra em relação à consistência dos dados cadastrais e número de entrevistados

\begin{tabular}{lcc}
\hline Característica da amostra & Número de pacientes & Porcentagem do total \\
\hline Número de telefone inválido & 154 & $43,26 \%$ \\
Não atenderam a ligação ou telefone desligado ou & 115 & $32,30 \%$ \\
fora de área & 30 & $8,43 \%$ \\
Paciente falecido & 57 & $16,01 \%$ \\
Pacientes que participaram da entrevista & 356 & $100,00 \%$ \\
\hline Total &
\end{tabular}

\section{Tabela 3}

Resultado das entrevistas por categoria

\begin{tabular}{llc}
\hline Categorias & Motivos & Número de repostas \\
\hline & Eu me esqueci da consulta & 3 \\
& A consulta coincidiu com outros compromissos & 4 \\
& Eu estava me sentindo muito mal para conseguir ir à consulta & 2 \\
Indivíduo/ & Eu não sentia mais os sintomas da doença para a qual iria me consultar & 1 \\
Comportamento & Eu consultei-me em outro lugar & 7 \\
& Falecimento & 0 \\
& Outros - Eu confundi a data da consulta & 2 \\
& Outros - Eu estava com depressão & 1 \\
& Outros - eu estava internado & 3 \\
& Outros - não me lembro & 4
\end{tabular}

(continua...) 
Tabela 3 (Continuação)

\begin{tabular}{llc}
\hline Categorias & Motivos & Número de repostas \\
\hline \multirow{3}{*}{ Família } & Outro membro da família estava doente & 0 \\
& Eu precisava buscar um familiar em outro local & 0 \\
Trabalho & Outros - Eu não tinha com quem deixar minha neta & 1 \\
Transporte & Não fui autorizado a sair do trabalho para ir à consulta & 2 \\
\multirow{2}{*}{ Clima } & Eu estava muito ocupado, precisava realizar muitas tarefas & 0 \\
Não havia transporte público que me levasse até o local da consulta & 1 \\
Outros & Não havia nenhuma pessoa para me conduzir até o local da consulta & 3 \\
& Estava chovendo muito no dia da consulta & 0 \\
\hline
\end{tabular}

Dos 57 pacientes entrevistados, 27 afirmaram ter faltado à consulta por motivos agrupados à categoria 1-Indivíduo/Comportamento, o qual apresentou como motivo mais frequente, a consulta ter sido realizada em outro lugar (7), seguido de horário e data da consulta ter coincidido com outro compromisso (4) e o paciente não se lembrar do motivo (4). O Esquecimento foi alegado por três pacientes, bem como estarem internados na data da consulta. Outros dois pacientes disseram estar se sentindo muito mal para conseguir comparecer à consulta, e dois pacientes confundiram a data da consulta. Apenas um paciente não sentia mais os sintomas da doença para a qual iria se consultar e um paciente disse não compareceu à consulta porque estava com depressão.

A categoria 2-Família contou apenas com um paciente que alegou não ter com quem deixar a neta, impossibilitando seu comparecimento à consulta. A categoria 3-Trabalho teve dois respondentes que alegaram não terem sido autorizados a sair ou faltar do trabalho para comparecer à consulta. A categoria 4-Transporte somou quatro razões, distribuídas entre a falta de acompanhante para conduzir o paciente até o local da consulta (3) e ausência de transporte público. Nenhum motivo foi relacionado a categoria 5-Clima.

Deve-se destacar que a categoria 6-Outros, teve 23 respondentes, dos quais dezoito alegaram não ter faltado à consulta e cinco disseram que nunca agendaram consulta no HCFMRP. Por conseguinte, a categoria 6-Outros, apresenta relevância frente ao número de causas de absenteísmo. Assim, devido ao elevado número de causas associadas a essa categoria e ao fato de todas elas estarem relacionadas a inconsistência de informações, foi possível criar uma nova categoria: "problemas ou falha de comunicação no processo". Além disso, como não houve razões relacionadas ao clima, esta categoria foi excluída da análise. De acordo com a nova categorização das respostas, as categorias mais relevantes foram "Indivíduo/Comportamento" $(n=27)$ e "Problemas de comunicação" $(n=23)$. Na Tabela 4 é apresentado os motivos das ausências distribuídos na nova categorização.

Tabela 4

Motivos distribuídos por categorias reajustado

\begin{tabular}{lcc}
\hline Categorias & Número de repostas & Porcentagem do total \\
\hline Indivíduo/Comportamento & 27 & $47,37 \%$ \\
Comunicação & 23 & $40,35 \%$ \\
Transporte & 4 & $7,02 \%$ \\
Trabalho & 2 & $3,51 \%$ \\
Família & 1 & $1,75 \%$ \\
\hline Total & 57 & $100,00 \%$ \\
\hline
\end{tabular}


A literatura evidencia que o absenteísmo sofre forte influência de questões ligadas ao paciente, como esquecimento, trabalho e família. $9,10,11,16$ No caso do HCFMRP, o motivo que merece destaque não está relacionado diretamente ao paciente, mas ao processo de agendamento. A categoria "Problemas de comunicação", com os motivos "Eu não faltei à consulta" e "Eu nunca tive uma consulta agendada no HCFMRP", contou com 23 respondentes. O desconhecimento do agendamento da consulta também foi identificado no estudo de Luckett et al. (2015) ${ }^{12}$ como uma causa importante do absenteísmo nos Estados Unidos. Esses resultados sugerem falhas no processo de agendamento. As informações sobre a consulta podem não ter sido transmitidas ao município ou, caso o município tenha sido informado, pode não ter transmitido a informação ao usuário. Erdmann et al. (2013) $)^{17}$ afirmam que problemas de comunicação nos municípios gera índice significativo de absenteísmo. Os problemas de comunicação limitaram o estudo, considerando que de 356 pacientes, apenas 57 foram entrevistados devido às falhas no processo de cadastro, com dados desatualizados ou inexistentes.

Como se trata de pacientes novos, essa faIha pode estar relacionada ao processo de acesso ao hospital, uma vez que as informações cadastrais são transferidas ao HCFMRP pelo município de origem do paciente. Assim, alguns dados podem estar incorretos porque foram transferidos erroneamente para a guia de referência no município e podem estar desatualizados devido ao longo período decorrido desde o preenchimento da guia de referência no SARA (momento da coleta dos dados do paciente na atenção básica/secundária) até o agendamento da consulta no hospital.

\section{CONCLUSÕES}

O presente trabalho foi realizado com o objetivo de analisar os motivos do absenteísmo dos novos pacientes nos ambulatórios oncológicos do Hospital das Clínicas da Faculdade de Medicina de Ribeirão Preto. Com base nos resultados obtidos, foi possível comparar as causas do absenteísmo no HCFMRP com àquelas encontradas na literatura. Conclui-se que, enquanto na maioria dos estu- dos publicados o absenteísmo é frequentemente relacionado a motivos ligados ao paciente, como esquecimento, trabalho e família, no caso estudado: pacientes oncológicos do HCFMRP, o motivo mais recorrente estava relacionado ao processo de agendamento. A categoria "Problemas de comunicação", com os motivos "Eu não faltei à consulta" e "Eu nunca tive uma consulta agendada no HCFMRP" foram predominantes entre os entrevistados, indicando a existência de falhas no processo de agendamento, já que pacientes que não estavam informados do agendamento da consulta constavam no relatório de faltas do hospital.

Outro resultado que deve ser destacado é a dificuldade para entrar em contato com os usuários: de um total de 356 pacientes, apenas 57 foram entrevistados com sucesso, o que indica falhas no processo de registro e atualização dos dados dos pacientes na rede de atenção à saúde. A ausência ou desatualização dos dados cadastrais dos usuários gerou a principal limitação deste estudo uma vez que impediu que o número amostral calculado fosse alcançado. Além disso, essa falha dificulta a implantação de estratégias para reduzir o absenteísmo, como ligações, mensagens e lembretes direcionados ao paciente para evitar o esquecimento da consulta. ${ }^{34}$ Assim, conclui-se que para resolver o problema do absenteísmo em consultas deve-se, inicialmente, buscar formas de manter os dados dos pacientes atualizados nos municípios e formas efetivas de compartilhamento de informação com as organizações que compõem a rede de atenção à saúde, uma vez que os problemas de cadastro do paciente e comunicação dificultam a compreensão das causas do absenteísmo e o desenvolvimento de ações para reduzi-lo.

\section{REFERÊNCIAS}

1. Mesa MLJ, Asencio JMM, Ruiz FR. Factores determinantes y coste económico del absentismo de pacientes em consultas externas de la Agencia Sanitaria Costa del Sol. An Sist Sanit Navar. 2015; 38:235-45.

2. Perron NJ, Dao MD, Kossovsky MP, Miserez V, Chuard C, Calmy A, Gaspoz JM. Reduction of missed appointments at an urban primary care clinic: a randomised controlled study. BMC Fam Pract. 2010;11:79. doi: 10.1186/1471-2296-11-79. 
3. Gurol-Urganci I, de Jongh T, Vodopivec-Jamsek V, Atun R, Car J. Mobile phone messaging reminders for attendance at healthcare appointments. Cochrane Database Syst Rev. 2013; (12):CD007458. doi: 10.1002/14651858. CD007458.pub3.

4. Hasvold PE, Wootton R. Use of telephone and SMS reminders to improve attendance at hospital appointments: a systematic review, J Telemed Telecare, 2011; 17: 358-64.

5. Zirkle MS, McNelles LR. Nonattendance at a hospital-based otolaryngology clinic: a preliminar analysis within a universal healthcare system. Ear Nose Throat J. 2011; 90:E32-4.

6. Neal RD, Hussain-Gambles M, Allgar VL, Lawlor DA, Dempsey O. Reasons for and consequences of missed appointments in general practice in the UK: questionnaire survey and prospective review of medical records. BMC Fam Pract. 2005;6:47.

7. Van der Meer G, Loock JW. Why patients miss follow-up appointments: a prospective control-matched study. East Afr J Public Health. 2008; 5:154-6.

8. Lehmann TN, Aebi A, Lehmann D, Balandraux Olivet $M$, Stalder, H. Missed appointments at a Swiss university outpatient clinic. Public Health. 2007;121:790-9.

9. Ferreira S, Freira S, Ferreira PD, Fonseca H. Faltas à consulta de adolescentes: motivos e dimensão do problema. Acta Pediátrica Portuguesa, 2011; 42: 258-63.

10. Nour El-Din MM, Al-Shakhs FN, Al-Oudah SS. Missed appointments at a university hospital in eastern Saudi Arabia: magnitude and association factors. J Egypt Public Health Assoc. 2008;83:415-33.

11. Guzek LM, Fadel WF, Golomb MR. A pilot study of reasons and risk factors for "No-Shows" in a pediatric neurology clinic. J Child Neurol. 2015; 30: 1295-9.

12. Luckett R, Pena N, Vitonis A, Bernstein MR, Feldman S. Effect of patient navigator program on no-show rates at an Academic Referral Colposcopy Clinic. J Womens Health. 2015; 24: 608-15.

13. Oleskovicz M, Oliva FL, Hildebrand e Grisi CC, Lima AC, Custódio I. Técnica de overbooking no atendimento público ambulatorial em uma unidade do Sistema Único de Saúde. Cad. Saúde Pública, Rio de Janeiro. 30:1009-1017.

14. Gonçalves CA, Vazquez FL, Ambrosano GMB, Mialhe FL, Pereira AC, Sarracini KLM, et al. Estratégias para o enfrentamento do absenteísmo em consultas odontológicas nas Unidades de Saúde da Família de um município de grande porte: uma pesquisa-ação. Ciênc. Saúde Colet. 20: 449-60.

15. Carvalho MAP, Sousa CMB, Lima Junior IA, Prado MF, Silva RNM. Estudo de caso sobre as faltas dos usuários às consultas programadas de uma equipe de saúde da família no município do Rio de Janeiro. J Manag Prim Health Care, 2014; 5: 140-52.

16. Izecksohn MMV, Ferreira JT. Falta às consultas médicas agendadas: percepções dos usuários acompanhados pela Estratégia Saúde da Família, Manguinhos, Rio de Janeiro. Rev Bras Med Fam Comunidade. Rio de Janeiro, 2014; 9:235-41. http://dx.doi.org/10.5712/rbmfc9(32)960.
17. Erdmann AL, Andrade SR, Mello ALSF, Drago LC. A atenção secundária em saúde: melhores práticas na rede de serviços. Rev Latino-Am Enfermagem, 2013; 21(Spec): [08 telas]

18. BRASIL. Ministério da Saúde. Portaria n. 4.279, de 30 de dezembro de 2010. Estabelece diretrizes para a organização da Rede de Atenção à Saúde no âmbito do Sistema Único de Saúde (SUS). Diário Oficial da União, Brasília, DF, dez. 2010.

19. Dallora MELV. Regulação do acesso dos usuários do SUS ao ambulatório de um hospital universitário (2010). Tese (Doutorado em Ginecologia e Obstetrícia) - Faculdade de Medicina de Ribeirão Preto, Universidade de São Paulo, Ribeirão Preto. Recuperado de http://www.teses.usp.br/ teses/disponiveis/17/17145/tde-19072010-134212/.

20. Silveira RP. Determinantes do absenteísmo nas consultas agendadas para a rede assistencial do Sistema Único de Saúde. Dissertação (Mestrado) - Faculdade de Medicina de Ribeirão Preto, Universidade de São Paulo, Ribeirão Preto. 2013.

21. Santos JS, Scarpelini S, Brasileiro ALL, Ferraz CA, Dallora MELV, Sá MFS. Avaliação do modelo de organização da unidade de emergência do HCFMRP-USP, adotado como referência, as políticas nacionais de atenção às urgências e de humanização da medicina. Medicina (Ribeirão Preto) 2003; 36: 498-515.

22. Lopes SL, Santos JS, Scarpelini S. The implementation of the Medical Regulation Office and Mobile Emergency Attendance System and its impact on the gravity profile of non-traumatic afflictions treated in a University Hospital: a research study. BMC Health Serv Res, 2007; 7: 173.

23. Ferreira JBB, Mishima SM, Santos JS, Foster AC, Ferraz $C A$. The regulatory complex for healthcare from the perspective of its operational players. Interface, Botucatu, 2010; 4: 345-58.

24. Santos JS. Protocolo para acesso aos Serviços do Sistema Único de Saúde. In: Santos JS, et al. (Orgs.). (2012). Protocolos clínicos e de regulação: acesso à rede de saúde. Rio de Janeiro: Elsevier. 2012; Cap. 1, 3-7.

25. Parikh A, Gupta K, Wilson AC, Fields K, Cosgrove NM, Kostis JB. The effectiveness of outpatient appointment reminder systems in reducing no-show rates. Am J Med. 2010;123:542-8. doi: 10.1016/j.amjmed.2009.11.022.

26. Mazza TO. Análise do absenteísmo de novos pacientes no Hospital das Clinicas da Faculdade de Medicina de Ribeirão Preto da Universidade de São Paulo; (Trabalho de Conclusão de Curso); (Graduação em Administração de Empresas) - Faculdade de Economia Administração e Contabilidade de Ribeirão Preto da USP. 2014.

27. Levine DM, Berenson ML, Stephan D. Estatística: teoria e aplicações usando Microsoft Excel em português. Rio de Janeiro: LTC, 2005.

28. Marconi MA, Lakatos EM. Técnicas de pesquisa: planejamento e execução de pesquisas, amostragens e técnicas de pesquisa, elaboração, análise e interpretação de dados. 7th ed. São Paulo: Atlas; 2008. 
29. Stevenson WJ, De Farias AA. Estatística aplicada à administração. Harbra. 1981.

30. Malhotra NK. Pesquisa de marketing: uma orientação aplicada. $4^{a}$ ed. Porto Alegre: Bookman;2006. p.720.

31. Almeida GL, Garcia LFR, Almeida TL, Bittar TO, Pereira AC. Estudo do perfil sócio-econômico dos pacientes e os motivos que os levaram a faltar em consultas odontológicas na estratégia de saúde da família em uma distrital de Ribeirão Preto/SP. Cienc Odontol Bras. 2009; 12: 77-86.
32. George A, Rubin G. Non-attendance in general practice: a systematic review and its implications for access to primary health care. Fam Pract. 2003;20:178-84.

33. Zailinawati $\mathrm{AH}, \mathrm{Ng} \mathrm{CJ}$, Nik-sherina $\mathrm{H}$. Why do patients with chronic illnesses fail to keep their appointments? A telephone interview. Asia Pac J Public Health. 2006;18:10-5.

34. Fischer $\mathrm{HH}$, Moore $\mathrm{S}$, Johnson $\mathrm{TL}$, Everhart RM, Batal $\mathrm{H}$, Davidsoni AJ. Appointment reminders by text message in a safety net health care system: a pragmatic investigation. EGEMS (Wash DC). 2017; 5:20. doi: 10.5334/egems.215. 